UCRL-JC- 126447

PREPRINT

\title{
Automatic vision system for analysis of microscopic behavior of flow and transport in porous media
}

\author{
Mehdi Rashidi \\ Jamshid Dehmeshki \\ Eric Dickenson \\ Farhang Daemi \\ This paper was prepared for submittal to the \\ SPIE Annual Meeting \\ San Diego, CA \\ July 27-August 1,1997
}

July 1997

This is a preprint of a paper intended for publication in a joumal or proceedings. Since changes may be made before publication, this preprint is made available with the understanding that it will not be cited or reproduced without the permission of the author. 


\section{DISCLAIMER}

This document was prepared as an account of work sponsored by an agency of the United States Government. Neither the United States Government nor the University of California nor any of their employees, makes any warranty, express or implied, or assumes any legal liability or responsibility for the accuracy, completeness, or usefulness of any information, apparatus, product, or process

disclosed, or represents that its use would not infringe privately owned rights. Reference herein to any specific commercial product, process, or service by trade name, trademark, manufacturer, or otherwise, does not necessarily constitute or imply its endorsement, recommendation, or favoring by the United States Government or the University of California. The views and opinions of authors expressed herein do not necessarily state or reflect those of the United States Government or the University of California, and shall not be used for advertising or product endorsement purposes. 


\title{
Automatic vision system for analysis of microscopic behavior of flow and transport in porous media
}

\author{
Mehdi Rashidi ${ }^{1}$, Jamshid Dehmeshki², Eric Dickenson ${ }^{1}$, and Farhang Daemi ${ }^{2}$ \\ 'Lawrence Livermore National Laboratory, University of California, Livermore, CA 94550, USA \\ ${ }^{2}$ Centre for Industrial and Medical Informatics, University of Nottingham, Nottingham, England
}

\begin{abstract}
This paper describes the development of a novel automated and efficient vision system to obtain velocity and concentration measurements within a porous medium. An aqueous fluid laced with a fluorescent dye or microspheres flows through a transparent, refractive-index-matched column packed with transparent crystals. For illumination purposes, a planar sheet of laser passes through the column as a CCD camera records all the laser illuminated planes. Detailed microscopic velocity and concentration fields have been computed within a 3D volume of the column. For measuring velocities, while the aqueous fluid, laced with fluorescent microspheres, flows through the transparent medium, a CCD camera records the motions of the fluorescing particles by a video cassette recorder. The recorded images are acquired automatically frame by frame and transferred to the computer for processing, by using a frame grabber and written relevant algorithms through an RS-232 interface. Since the grabbed image is poor in this stage, some preprocessings are used to enhance particles within images. Finally, these enhanced particles are monitored to calculate velocity vectors in the plane of the beam. For concentration measurements, while the aqueous fluid, laced with a fluorescent organic dye, flows through the transparent medium, a CCD camera sweeps back and forth across the column and records concentration slices on the planes illuminated by the laser beam traveling simultaneously with the camera. Subsequently, these recorded images are transferred to the computer for processing in similar fashion to the velocity measurement. In order to have a fully automatic vision system, several detailed image processing techniques are developed to match exact images (at different times during the experiments) that have different intensities values but the same topological characteristics. This results in normalized interstitial chemical concentrations as a function of time within the porous column.
\end{abstract}

Keywords: imaging, flow, transport, vision systems, image processing, porous media, fluorescent techniques

\section{INTRODUCTION}

Porous flow technology plays an important role in the chemical, biological, and environmental process industries. Systems involving a porous medium are common in areas from the remediation of chemicals in subsurface to the production of food, pharmaceuticals, and specialty materials. Despite wide occurrence of such systems, our understanding of these processes are still based on intuition and correlations rather than on fundamentals. The principal reason is that the controlling pore-scale flow regimes are very complex and the relation between the microscale and macroscale flow regimes is not well-understood. The lack of detailed microscale information brings about the need for novel automated imaging systems that can unravel the controlling pore-scale transport mechanisms and help researchers in modeling these processes.

Previous experimental studies have provided some macroscopic results but limited microscopic data on the chemical flow and transport in porous media. Experiments of Schwartz and Smith ${ }^{1}$, Murphy ${ }^{2}$, and Musser ${ }^{3}$ in packed beds have shown the development of a peak in velocity profile very close to the wall. However, these results were not characterized conclusively as a function of the flow Reynolds number (dimensionless number characterizing the flow: ratio of inertial effects to viscous effects) and the pore geometry. Flow visualization and transport studies of Joll and Hanratty ${ }^{4-5}$ revealed some flow and mass transfer information near spherical particles. Joll and Hanratty ${ }^{4}$ examined the path line of flow through a packed bed of spherical particles using dye visualization. They observed a transition from laminar to turbulent flow at Reynolds numbers of 268 to 366 (based on pore velocity and particle diameter). Furthermore, they observed a smooth transition from steady to unsteady mass transfer at approximately the same Reynolds numbers.

Experimental studies of Harleman and Rumer ${ }^{6}$, Hassinger and Von Rosenberg ${ }^{7}$, Klotz et al..$^{8}$, and Han et al.$^{9}$ have provided some macroscopic estimates of longitudinal and lateral dispersion coefficients. Han et al..$^{9}$ studied the effect of column length and particle size distribution on the two components of the dispersion coefficients. They showed that for the case of uniform size particles, the longitudinal dispersion coefficients were a strong function of axial position in the bed unless the dispersion length satisfied a constraint dependent on the value of the Peclet 
number (dimensionless number characterizing the transport: ratio of dispersive/convective effects to diffusive effects). Furthermore, it was seen that for the case of the wide size particle distribution, longitudinal dispersion coefficients were larger (up to 3 times) than in the uniform size particle distribution case, and a longer dispersion length was required in order to obtain a constant dispersion coefficient. However, due to the limited amount of data, they could not provide a quantitative measure of the size of this length scale.

Laser anemometry and flow visualization study of Dybbs and Edwards ${ }^{10}$ for liquid flows in several porous structures has shown the existence of four flow regimes as a function of flow Reynolds number (based on particle diameter and pore velocity). These include a Darcy flow regime $(\operatorname{Re}<1)$, an inertial flow regime $(1<\operatorname{Re}<150)$, an unsteady laminar flow regime $(150<\operatorname{Re}<300)$, and a turbulent flow regime $(\operatorname{Re}>350)$. Their results also showed an increase in velocity profile in about one to two particle diameters from the wall, similar to the findings of Schwartz and Smith ${ }^{1}$.

Although these experimental efforts have provided some valuable information on the macroscopic behavior of the flow and transport in porous media, until recently, little work has been done on the microscopic characterization of processes at the pore-scale. This can be attributed to the experimental difficulty of nonintrusively measuring flow and transport at high resolutions within the solid matrix. Recent experimental improvements have allowed some renewed investigation of pore-scale processes. These include studies using certain forms of noninvasive imaging techniques (i.e., PIV, PVI, and MRI) in and above packed beds for velocity, porosity, and phase distribution measurements ${ }^{11-17}$ and studies in surrogate media composed of $2 \mathrm{D}$ etched glass or capillary network micromodels with known pore geometries ${ }^{18-20}$.

Theoretical descriptions of flow and transport in porous media have been generally derived from simpler "bulk" equations of mass and momentum balance or from more systematic approaches in which pore-scale behavior is rigorously averaged over the representative elementary volume (REV) of the medium. The averaging process introduces new terms that have to be characterized through systematic microscopic experiments.

The present work is part of an extensive research in our laboratories to understand the nature of the microscopic flow and transport processes within porous media. A novel nonintrusive imaging approach has been used to observe the pore-scale flow and transport behavior at a high resolution and a high accuracy ${ }^{21}$. Previously, we applied these techniques to study flow and solute transport within a non-aqueous homogeneous porous system ${ }^{22-23}$. This paper reports on the successful application of our experimental imaging techniques using our automated image processing system for transport studies in aqueous heterogeneous porous systems ${ }^{24}$. Furthermore, it provides some velocity and concentration results on flow experiments performed in our unique refractive index-matched aqueous systems with natural packing minerals of similar shape and sizes as to soil particles. The overall objective is to use these findings toward gaining a physical understanding of porous flow regimes and, as a result, provide the basis for improved modeling of these flow processes.

\section{MODELING APPROACHES}

Traditionally, there have been three different approaches to the modeling of flow and transport in porous media. The first approach is a statistical method treating the porous media as a random structure. The second is a geometrical approach assuming a similar known geometry to the porous media under study in order to solve the conservation equations. The third approach (continuum) is a volume averaging procedure that allows one to describe porous medium transport in terms of macroscopic rather than microscopic scale variables. All three approaches result in undetermined parameters that must be evaluated from experiments. However, the third approach (volume averaging method), if done correctly, provides the most general macroscopic conservation equations for transport in porous media. The volume averaging or continuum approach is the basis for the most recent theoretical investigations. Here, the general microscopic equation of transport in porous media

$$
\frac{\partial \rho \psi}{\partial \mathrm{t}}+\nabla \cdot(\rho \mathbf{v} \psi)-\nabla \cdot \mathbf{J}-\rho \mathrm{f}-\rho \mathrm{G}=0
$$

where,

$\rho$ is the mass density function,

$\psi$ is a typical intrinsic thermo-mechanical property,

$\mathbf{v}$ is a velocity vector,

$\mathbf{J}$ is a flux term,

f is an external supply term, and

$G$ is the net rate of production. 
is volume averaged over a representative elementary volume (REV) of the medium. Here, the intricate structure of pore networks and grain boundaries are replaced with an effective continuum and an averaged set of variables and balance equations. These equations are derived from their pore-scale counterparts by the method of volume averaging. The works of Whitaker ${ }^{25}$, Slattery ${ }^{26}$, Bear ${ }^{27}$, Hassanizadeh and Gray ${ }^{28}$, Gray et al. ${ }^{29}$, and Rashidi ${ }^{30}$ provide some insights of the current approach in this field. While each model presents a slightly different point of view, all require some assumptions about a specific medium behavior that must be confirmed and parameterized by detailed experimental work.

\section{EXPERIMENTAL FACILITIES AND TECHNIQUES}

Experiments in the aqueous system were performed in a clear Plexiglas rectangular packed column $3.0 \mathrm{~cm}$ in width and about $22.0 \mathrm{~cm}$ in length. Figure 1 shows the experimental setup and the orientation of measurement devices. The difficulty in performing experiments in aqueous system was to be able to find optically clear materials that have refractive indices close to water's refractive index of about 1.33 at $20.0^{\circ} \mathrm{C}$ and $514.5 \mathrm{~nm}$. This task was accomplished after many searches and tests. Some natural minerals were discovered that are found in nature or made as crystals in the laboratory with refractive indices close to that of water. These minerals are the crystals of Lithium fluoride salts $(\mathrm{LiF})$ that were made in the laboratory to the range of size specification for these experiments. Therefore, the column was filled with these natural mineral particles of nonuniform sizes with average diameters of about $0.15 \mathrm{~cm}$. An aqueous solution was chosen as the fluid which matched the particles' refractive index at $20.0^{\circ} \mathrm{C}$ and a wavelength of $514.5 \mathrm{~nm}$. The refractive index of the aqueous solution was matched to the refractive index of the particles by adjusting the temperature and small addition of dissolved sugar (sucrose) to water. The refractive index matching was critical up to third digit after decimal point, for matching the refractive indices of the packing particles and the aqueous solution. The column was maintained at this temperature throughout all runs by being immersed in a circulating constant temperature bath. A constant volumetric flow rate of the above fluid was provided for each run covering a wide range of Reynolds number, $\sim 10^{-3}$ to 1 (Reynolds number is based on average pore velocity and average particle diameter).

The experimental setups were designed such that, at the test section, flow was free from any side wall or entrance effects. The experiments were done with the refractive matched fluid seeded with fluorescent latex microspheres of 3 $\mu \mathrm{m}$ in diameter (for velocity measurements) or an organic fluorescent dye (for concentration measurements). The column was illuminated by an Argon-ion laser (coherent) operated at $475 \mathrm{~nm}$ for velocity measurements and $488 \mathrm{~nm}$ for concentration measurements. A CCD camera was used to record the experimental runs. Since the dye emission peaks around $514.5 \mathrm{~nm}$, a band pass filter was used on the video camera to pass a narrow range of $514.5 \mathrm{~nm} \pm 5 \mathrm{~nm}$ wavelength associated with the dye excitation. The video camera was placed perpendicular to the laser propagation beam on a remotely operated platform such that it moved with the beam in order to scan various cross-section of the column.

As shown in Figure 2, the refractive index-matching yields a transparent porous medium, free from any scattering and refraction at the solid-liquid interfaces, thus allowing direct optical probing at various vertical planes within the porous system. In these experiments, first the velocity measurements were taken by sequentially scanning the flow field in vertical cross-sections. Velocity measurements were obtained by tracking the seeded microspheres (particle density $\approx 10$ particles $/ \mathrm{mm}^{3}$ ) in the fluid. This provided velocity measurements of the longitudinal and one transverse velocity components at a large number of microscopic locations within the cylindrical test section. Then, a neutrally buoyant organic dye (molecular diffusivity, $D_{m} \approx 10^{-5} \mathrm{~cm}^{2} / \mathrm{sec}$ ) was steadily introduced into the column and its concentration was imaged within the same segment of the column, where the velocity field was determined. The video camera recorded fluorescence images on a series of vertical planes while sweeping back and forth (with the illumination plane) across the column once every minute. This allowed interstitial chemical concentrations to be determined as a function of time in the same planes where velocity measurements were taken. Roughly ten seconds were required to sweep through all 60 vertical planes within the column. Sequential sweeps were separated by thirtysecond intervals. Measurements from each sweep were collected into aggregate blocks of data and used to approximate a snapshot of the system at a single point in time. Since the flow was slow enough and the plane to plane time delay was significantly shorter than the time between complete sweeps through the volume, concentration images of vertical planes in one sweep could be considered to be made simultaneously. Dye concentration measurements also allowed the pore geometry on each plane to be measured. These images were recorded through a high resolution video camera by a frame accurate VCR and then analyzed rigorously using an IBM compatible computer.

The experimental runs are recorded through the video camera by a computer controlled VCR (Sony, EVO-9650) in Hi-8 mm format. The VCR is a frame accurate model that produces high quality still images of specified frames. It is computer controlled through an RS-232 interface for automated velocity and concentration analysis. In order to 


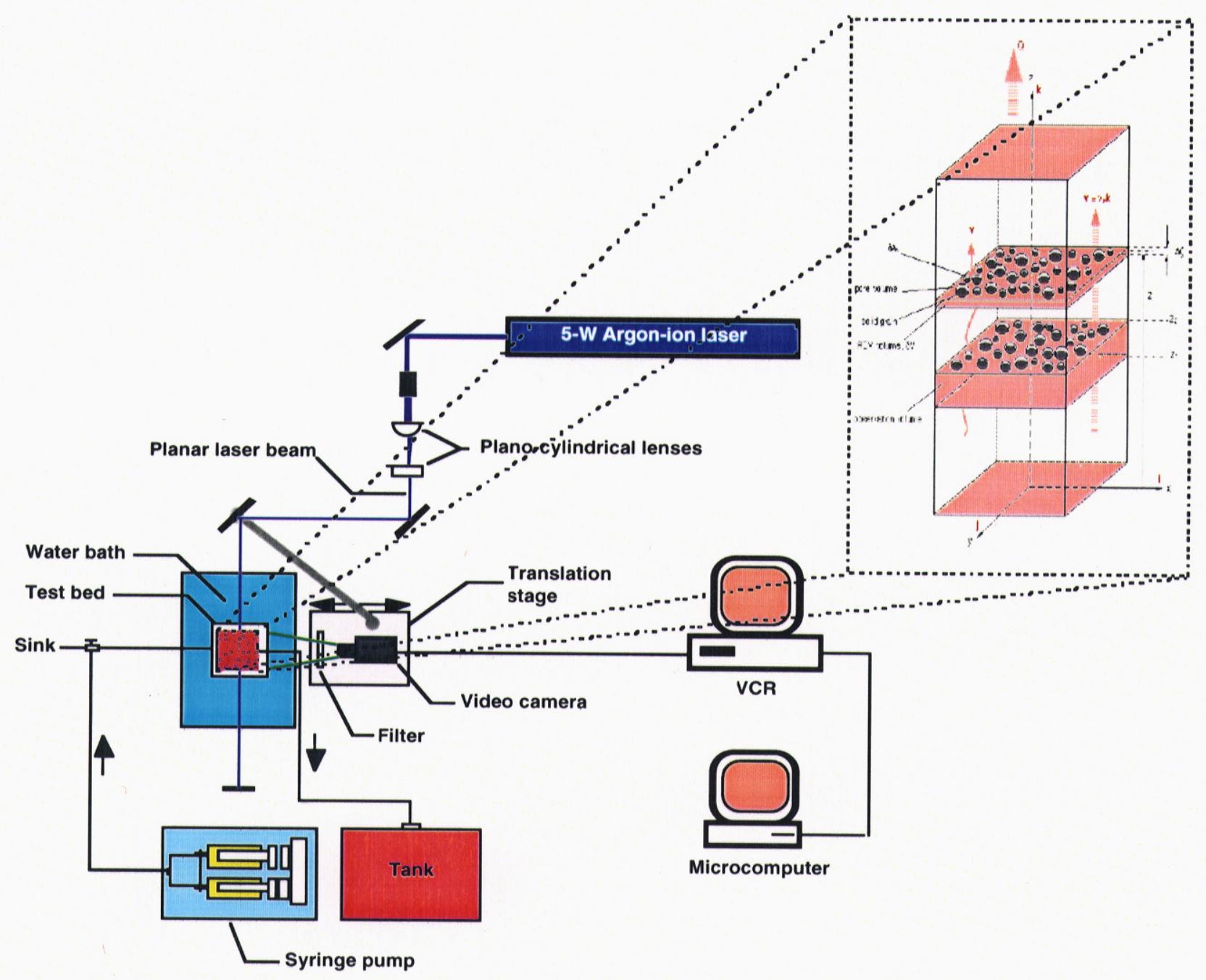

Figure 1. Experimental setup and measurement techniques.
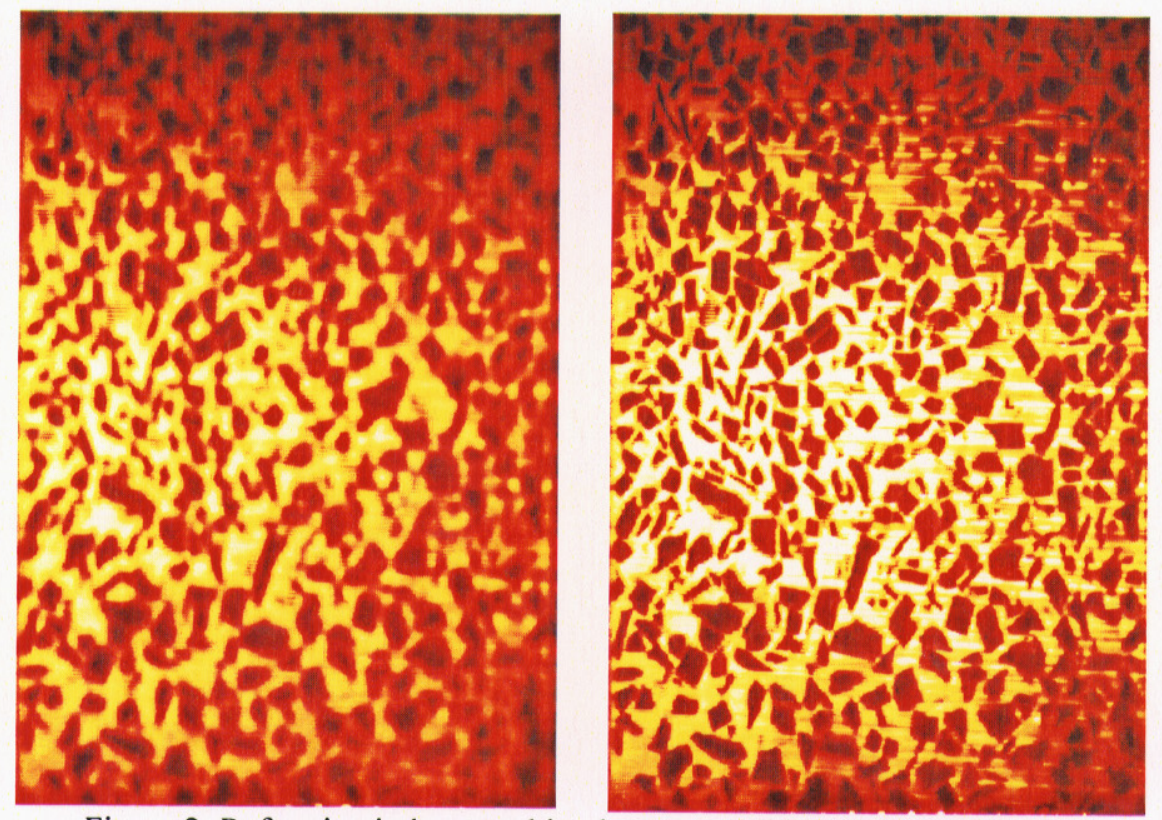

Figure 2. Refractive index matching between solid particles and liquid. Left picture: not matched; Right picture: matched 
capture the recorded images for data analysis, two image processing systems are used. One system is utilized for concentration analysis. For this system, a frame grabber board (ITI PC Vision Plus) with 640 by 480 pixel resolution is used in conjunction with an IBM compatible 486-133 MHz computer. The other faster system is used for velocity analysis and has a PCI frame grabber (Coreco TCI-VGA 2 Ultra) board at $640 \times 480$ pixel resolution with an IBM compatible PentiumPro $200 \mathrm{MHz}$ computer. The image analysis is done using the OPTIMAS (BioScan) and the AIDA (our laboratory's developing) softwares. Several detailed programs have been developed in our laboratories as interface softwares, for automated experimental run and analysis of velocity and concentration measurements.

Experiments were conducted in the described facilities under seven flow conditions as summarized in Table I. The details of the experimental conditions are summarized in Table I. They were performed at seven different Reynolds (and Peclet) numbers with the Reynolds numbers being based on the average packing particle diameter and pore velocity. As a result, a wide range of flow conditions were studied in these experiments.

Measurement uncertainties were evaluated for all the runs. The uncertainty in the values of average axial velocity was about $2 \%$ at $95 \%$ confidence level for 200 frames analyzed (see Rashidi and Banerjee ${ }^{31}$, for details on uncertainty analysis of particle tracking techniques). Similarly, the concentration and porosity measurements revealed an uncertainty estimate of about $2 \%$ at $95 \%$ confidence level. Rashidi ${ }^{30}$ provides more details on the image analysis techniques and the comparison of the three widely used velocimetry techniques of PIV, PSV, and PTV. The present technique (PTV) provides an improvement in measurement uncertainties and resolution over our previous experiments.

Table I. Experimental Conditions.

\begin{tabular}{|c|c|c|c|c|c|c|c|c|}
\hline Run & $\begin{array}{c}\mathrm{T} \\
\left({ }^{\circ} \mathrm{C}\right)\end{array}$ & $\begin{array}{l}\text { Fluid } \\
\text { Kinematic } \\
\text { Viscosity } \\
\left.\text { v ( } \mathrm{cm}^{2} / \mathrm{s}\right)\end{array}$ & $\begin{array}{c}\text { Average } \\
\text { Column } \\
\text { Porosity } \\
\phi\end{array}$ & $\begin{array}{l}\text { Volumetric } \\
\text { Flow } \\
\text { Rate } \\
\mathrm{Q}\left(\mathrm{cm}^{3} / \mathrm{min}\right)\end{array}$ & $\begin{array}{l}\text { Average } \\
\text { Pore } \\
\text { Velocity } \\
\overline{\mathrm{v}}(\mathrm{cm} / \mathrm{s})\end{array}$ & $\begin{array}{l}\text { Average } \\
\text { Particle } \\
\text { Diameter } \\
\mathrm{d}_{\mathrm{p}}(\mathrm{cm}) \\
\end{array}$ & $\begin{array}{l}\text { Reynolds } \\
\text { Number } \\
\text { Re } \\
\left(\bar{v} d_{p} / v\right)\end{array}$ & $\begin{array}{c}\text { Peclet } \\
\text { Number } \\
\text { Pe } \\
\left(\overline{\mathrm{v}} \mathrm{d}_{\mathrm{p}} / \mathrm{D}_{\mathrm{m}}\right)\end{array}$ \\
\hline 1 & 20.0 & 0.0381 & 0.4 & 0.23 & 0.0011 & 0.15 & 0.0040 & 4.8 \\
\hline 2 & 20.0 & 0.0381 & 0.4 & 0.57 & 0.0027 & 0.15 & 0.010 & 12 \\
\hline 3 & 20.0 & 0.0381 & 0.4 & 1.2 & 0.0053 & 0.15 & 0.020 & 24 \\
\hline 4 & 20.0 & 0.0381 & 0.4 & 2.3 & 0.011 & 0.15 & 0.040 & 48 \\
\hline 5 & 20.0 & 0.0381 & 0.4 & 5.7 & 0.027 & 0.15 & 0.10 & 121 \\
\hline 6 & 20.0 & 0.0381 & 0.4 & 12 & 0.055 & 0.15 & 0.20 & 251 \\
\hline 7 & 20.0 & 0.0381 & 0.4 & 23 & 0.11 & 0.15 & 0.40 & 482 \\
\hline
\end{tabular}

\section{VELOCITY MEASUREMENT TECHNIQUES}

\subsection{Image acquisition}

While the aqueous fluid, laced with fluorescent microspheres, flows through the transparent medium, the computer controlled CCD camera rasters across the width and height of the column for each planar slice, scans and records velocity images on the illuminated planes using the frame-accurate video cassette recorder (VCR). These recorded images are transferred to the computer for processing, by using a frame grabber and written relevant algorithms through a RS-232 interface.

\subsection{Preprocessing stage}

Since in the grabbed images, some microspheres are not clearly distinguished (specially in the slices farther away from the camera), some preprocessings are done to enhance the images being studied. Figure $3 \mathrm{a}$ shows a typical image sample of microspheres flowing in the column. A sharpening filter is applied to the image in Figure 3a to detect the sharp edges of the microspheres. The convolution mask used in the sharpening filter has a positive coefficient in its center and mostly negative coefficients around the outer edge. It uses a high pass filter mask, which removes the low frequency component and shows only high details. This convolution is a high pass $5 \times 5$ filter that 
specifically sharpens edges and enhances details. This filter causes magnification of gradients along edges in any direction. The kernel is shown below:

$\begin{array}{rrrrr}-1 & -1 & -1 & -1 & -1 \\ -1 & 1 & 1 & 1 & -1 \\ -1 & 1 & 9 & 1 & -1 \\ -1 & 1 & 1 & 1 & -1 \\ -1 & -1 & -1 & -1 & -1\end{array}$

Figure $3 \mathrm{~b}$ shows the result of the applied sharpening filter. Then, the image is binarized using a certain threshold to separate the foreground from the background. Binarizing distinguishes which pixels are microspheres, as shown in Figure 3c. Now microspheres are more readily trackable and velocities can be determined with much higher accuracy.

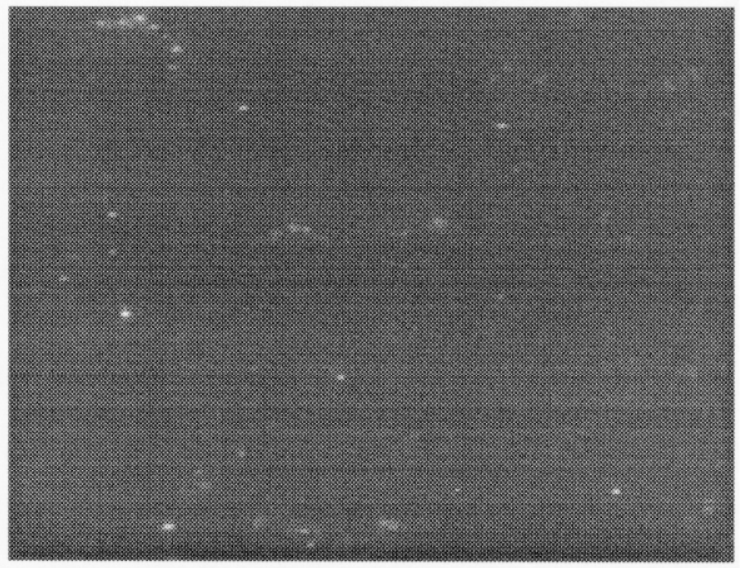

Figure 3a. Typical image sample of microspheres flowing in the column.

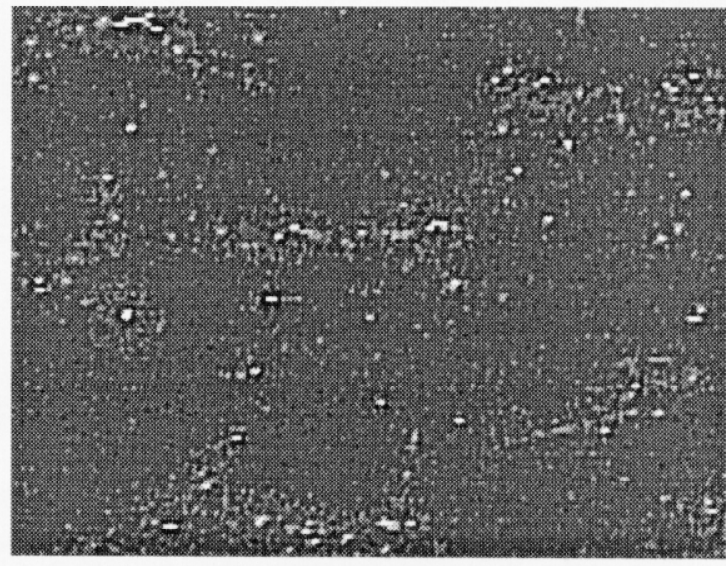

Figure 3b. Sharpening filter applied to a typical image to enhance edges of microspheres.

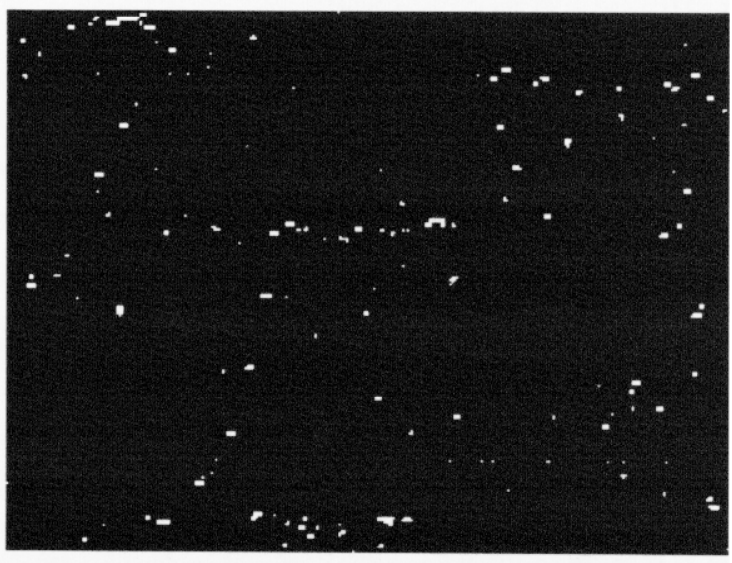

Figure 3c. Image in Figure $3 b$ is binarized. Microspheres are more easily detectable and trackable. 


\subsection{Velocity Measurement Results}

Two components of the velocity are observed in the plane of the light. Denoting the light sheet as the $x-z$ plane, one can determine $x$ - and $z$ - velocities over the range of the $x$ - and $z$-positions in the field-of-view. Here, $z$ axis is the axial coordinate in the flow direction along the column length and $x$ axis is in the transverse direction. The Particle Tracking Velocimetry (PTV) technique is used to analyze and calculate velocities. In this particle tracking algorithm all of the microspheres in the first frame are located and their positions are recorded. Then the tape is advanced one or several frames (depending on the flow rate), and each microsphere is relocated by using the previous microsphere position and the new position is recorded. The microsphere's velocity is calculated by fitting the time history of its path, $x(t)$ and $z(t)$, using linear regression. Figure 4a shows a collection of velocity vectors (over $0.5 \mathrm{~mm} \times 0.5 \mathrm{~mm}$ areas) in one field-of-view, which were produced from the experimental run with an injection volumetric flow rate of $5.73 \mathrm{~cm}^{3} / \mathrm{min}$. Velocity scale is represented by the narrow head segments, which have a length scale corresponding to $20 \mu \mathrm{m} / \mathrm{s}$. The largest velocity is about $780 \mu \mathrm{m} / \mathrm{s}$ and the smallest velocity is $0 \mu \mathrm{m} / \mathrm{s}$ (some particles did not move). These results represents the motion of about 350 microspheres over the course of 150 frames. Since the flow is at steady state, to increase our measurement accuracy and to reduce the quantity of data, the velocity vectors are averaged over $0.5 \mathrm{~mm} \times 0.5 \mathrm{~mm}$ areas forming a grid, as shown in Figure $4 \mathrm{~b}$. In these figures, the clear areas with no arrows are where the solid packing particles exist in the column, therefore there is no flow through these areas.

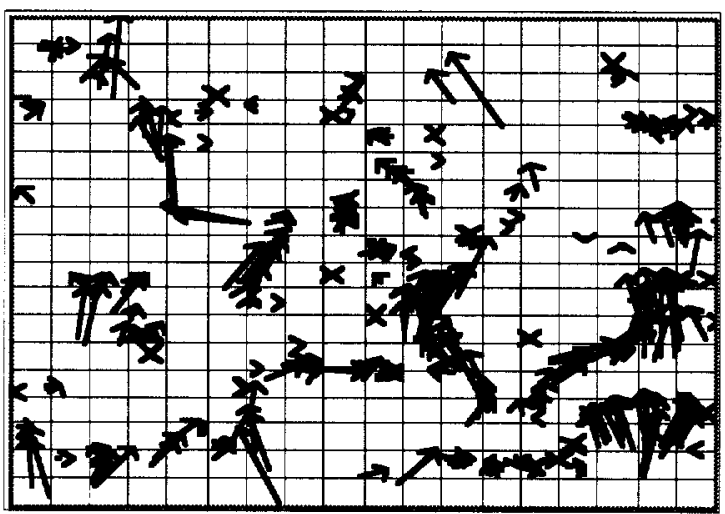

Figure 4a. Particle velocity vectors calculated from 150 flow images. Field of view $=9 \mathrm{~mm} \times 9 \mathrm{~mm}$. The arrow head segments $=20 \mu \mathrm{m} / \mathrm{s}$. Individual velocity values range from 0 to $783 \mu \mathrm{m} / \mathrm{s}$.

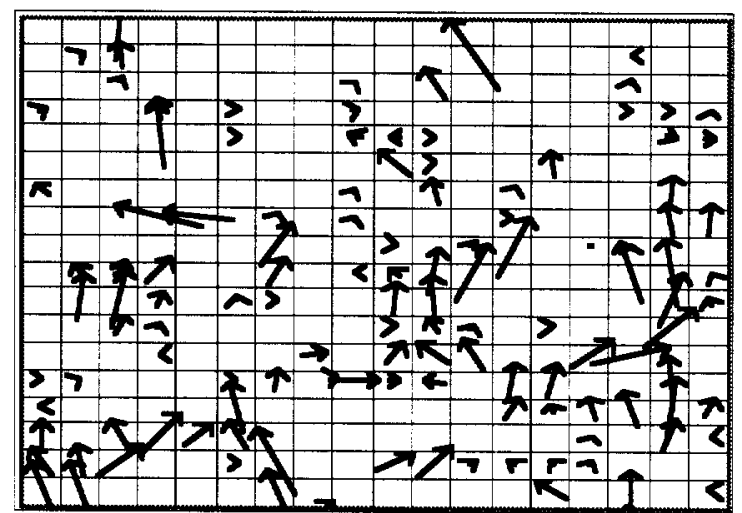

Figure $4 \mathrm{~b}$. Same region in Figure $4 \mathrm{a}$ and it shows averaged velocities over $0.5 \mathrm{~mm} \times 0.5 \mathrm{~mm}$ areas to form a grid.

\section{CONCENTRATION MEASUREMENT TECHNIQUES}

\subsection{Image acquisition}

As the aqueous fluid, laced with fluorescent dye, flows through the transparent medium, the CCD camera sweeps back and forth across the column and records concentration images on the illuminated planes using the frameaccurate video cassette recorder (VCR). Once again, these recorded images are transferred to the computer for processing, by using a frame grabber and written relevant algorithms through a RS-232 interface.

\subsection{Preprocessing stage}

Before images are processed, since experiments are performed dynamically and a given slice has to be analyzed at different time intervals throughout the tape, the images with the same solid phase topology and differing intensities must be matched automatically. Varying intensity images are matched with the saturated image of identical solid phase topology. The approximated location on a recorded tape of the image to be matched is known. Four adjacent candidate images or frames are analyzed, compared, and matched to the saturated image of the same slice. The saturated image is shown in Figure 5a. First a sharpening filter is applied to the saturated image and to the four adjacent images (as matching candidates) to emphasis the solid phase's sharp edges (as described in velocity measurement section 4.2). Figure $5 \mathrm{~b}$ shows the result of the applied sharpening filter to the saturated image. Since the high pass filter mask, in the sharpening filter, enhances fine detail in an image, it also tends to amplify the noise in an image. An effective method for removing noise is the application of a median filter. Median filtering is a nonlinear area process that has been researched extensively. One great advantage of the median filter over the low pass filter is the median filter does a much better job of preserving sharp edges of the image. This is important, 
since it is crucial in preserving the exact topology of the solid phase. Figure $5 \mathrm{c}$ is the result of the use of a median filter on Figure $5 \mathrm{~b}$. This image shows the median filtering forces points with very distinct intensities to be more like their neighbors, thus eliminating intensity spikes. Then, the image is binarized using a certain threshold, as shown in Figure $5 \mathrm{~d}$ for the saturated image and in Figure 5e for one of the four candidate images to be compared to. Then, a XOR function is applied which compares each pixel of the saturated image with each corresponding pixel of the four candidate images by calculating the absolute value of the difference of the pixel values. For example, this is performed on the images in Figures $5 \mathrm{~d}$ and $5 \mathrm{e}$ and performed on the image in Figure $5 \mathrm{~d}$ and on the rest of the 3 images to be compared. Figures $5 \mathrm{f}, \mathrm{g}, \mathrm{h}$, i show the result of the XOR function on the four images. Then, the percent white area is calculated. The percent white areas of $16.3 \%, 11.8 \%, 14.9 \%, 20.8 \%$ are determined for Figures $5 \mathrm{f}, 5 \mathrm{~g}$, $5 \mathrm{~h}$, and $5 \mathrm{i}$, respectively. Figure $5 \mathrm{~g}$ has the lowest white area percentage of $11.8 \%$ and best matches the saturated image's solid phase topology. Therefore, this image is picked for concentration analysis at the given time.

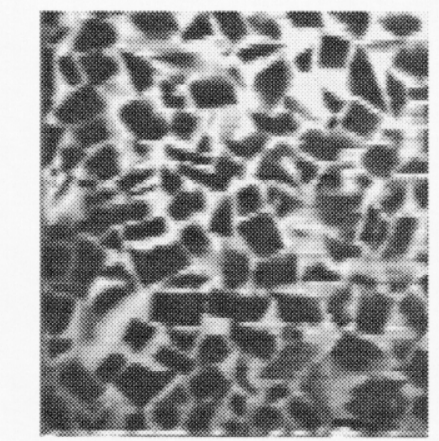

Figure 5a. Image slice after saturation of dye.

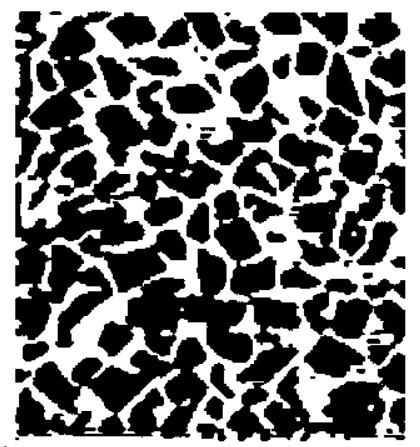

Figure $5 \mathrm{~d}$. The saturated image of Figure $5 \mathrm{c}$ is binarized.

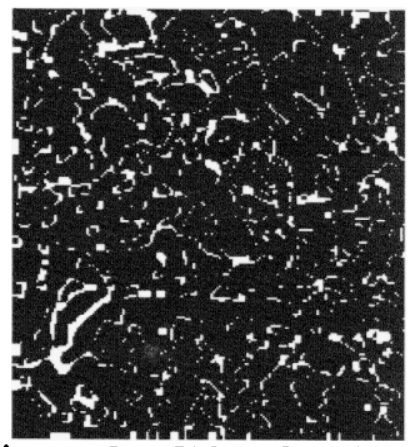

Figure $5 \mathrm{~g}$. XOR function is applied to saturated and second candidate image out of four comparing images.

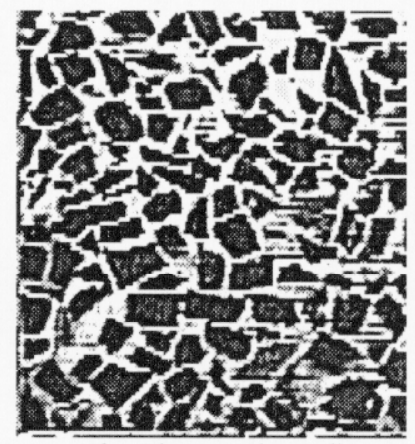

Figure 5b Sharpening filter is applied to saturated image of Figure 5a.

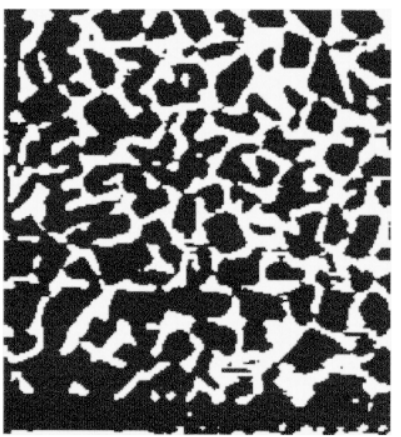

Figure 5e. One of the four images to be compared, after the application of a sharpening and median filters and binarization.

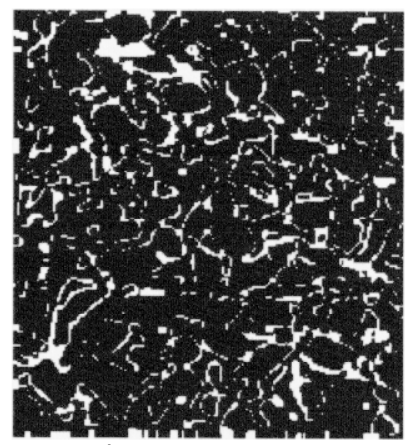

Figure $5 \mathrm{~h}$. XOR function is applied to saturated and third candidate image out of four comparing images.

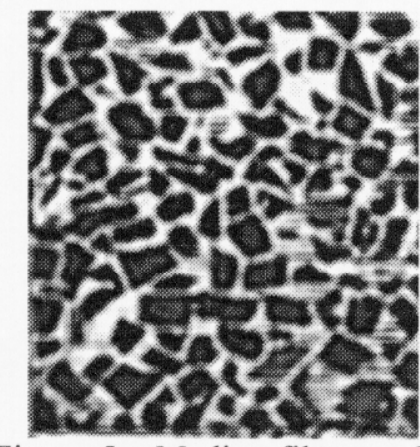

Figure 5c. Median filter applied to saturated image of Figure 5b.

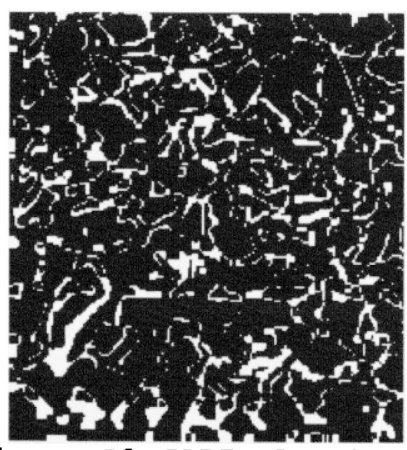

Figure 5f. XOR function is applied to saturated and first candidate image (Figure $5 \mathrm{~d}$ ) out of four comparing images.

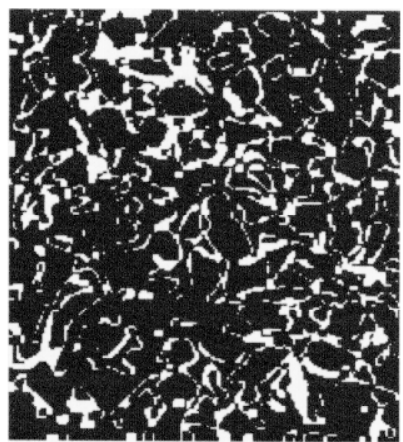

Figure 5i. XOR function is applied to saturated and fourth candidate image out of four comparing images. 
Since the grabbed images are poor in this stage, some preprocessings are done to enhance the images under study. Due to a nonuniform illumination problem of the laser plane, the intensities of pixels values of recorded images are higher on the left side of the image. Figure 6a shows a typical image sample at a couple of minutes after dye has been introduced to the column and it shows the intensities of pixel values are higher on the left side of the image. This nonuniform problem can be seen in the histogram of Figure 6a, as shown in Figure 7a. The vertical bars of the histogram are tightly clustered together and a low range of all the possible pixel values is used. A Contrast Stretching technique works best on these images since most of the pixels are concentrated in one part of the histogram. It can be applied to the image to stretch the histogram to fill the full dynamic range of the image.

Contrast Stretching is a useful technique to enhance images that have low contrast, such as the image shown in Figure 6a. The resulting image exhibits a wide range of pixel values and no single shade dominates the image. The following equation is the basis for the Contrast Stretching technique:

$$
\text { NewPixelValue }=\frac{(\text { CurrentPixelValue }- \text { LowPixelValue })}{(\text { HighPixelValue }- \text { LowPixelValue })} \times 255
$$

Here, the Low and High Pixel Values are the images with no dye concentration (Figure 6b- black image) and with the highest dye concentration (Figure 6c- saturated image), respectively. These images have varying dye concentration intensity values, but they have the same exact solid phase topology. In equation (2), when the Low Pixel Value (Figure 6b) is subtracted from Current Pixel Value (Figure 6a), the histogram in Figure 7a will slide to the left, as shown in Figure $7 \mathrm{~b}$. The lowest pixel value is now zero. When the nominator is divided by the denominator in equation (2), each pixel value is scaled so that the image fills the entire dynamic range. The result is an image that spans the pixel values from the Low Pixel Value (black image) to High Pixel Value (saturated image), as shown in Figure $6 \mathrm{~d}$. Figure $7 \mathrm{c}$ shows the histogram of Figure $6 \mathrm{~d}$. Figure $6 \mathrm{~d}$ shows that noise was introduced in the solid phase. In these areas there are false and meaningless pixel values, which are converted to zero by multiplying the image in Figure $6 \mathrm{~d}$ by the Bead image (Figure $6 \mathrm{~g}$ ).

The Bead image is a binarized image that has the same topology of the image being analyzed. The Bead image is formed by first subtracting the Low Pixel Value image (Figure 6b) from High Pixel Value image (Figure 6c) and then applying the sharpening filter to increase the image's contrast and emphasis details, such as solid phase's sharp edges. Figure 6e shows the result of the applied sharpening filter. Since the high pass filter mask, in the sharpening filter, enhances fine details in an image, it also tends to amplify the noise in an image. An effective method for removing noise is the application of a median filter which also does a good job of preserving sharp edges of the image. This is important in our Bead image, since it is crucial in preserving the exact topology of the solid phase. Figure $6 \mathrm{f}$ is the result of the use of a median filter on Figure 6e. This image shows the median filtering forces points with very distinct intensities to be more like their neighbors, thus eliminating intensity spikes. Then the Bead image is binarized using a certain threshold, as shown in Figure $6 \mathrm{~g}$. Finally, Figure $6 \mathrm{~h}$ shows the result of multiplying the normalized image, Equation (2) (Figure 6d), by the Bead image (Figure 6g). Figure 6h shows that pixel values in the solid phase have been successfully converted to 0 values.

\subsection{Concentration measurement results}

As shown in Table I, seven different experimental runs were performed using the described facilities and imaging techniques. For the seven different injection volumetric flow rates of solute, nondimensional cross-sectional average concentrations were estimated between axial positions $\mathrm{z}=0 \mathrm{~cm}$ and $\mathrm{z}=3.2 \mathrm{~cm}$. The average concentrations in Figures 8 and 9 are produced from an injection volumetric flow rate of $5.7 \mathrm{~cm} 3 / \mathrm{min}$ (Run 5). Figure 8 shows the average concentration as a function of time at selected locations along the z-axis. As shown here, the column gets saturated in about 15 minutes after the start of this run. These plots show the typical breakthrough curves obtained in saturation experiments in porous media. Figure 9 shows the average concentration as a function of axial position within the column at several different times. These plots provide the concentration gradients throughout the column at different time for this experimental run. Similar breakthrough curves are produced from other experimental runs with injection flow rates of $0.23,0.57,1.2,2.3,12$ and $23 \mathrm{~cm} 3 / \mathrm{min}$. Figure 10 shows the volumetrically averaged concentration as a function of time for the seven different injection flow rates. As seen here, the column gets saturated with the contaminant solute in less than 5 minutes for $\operatorname{Run} 7(\operatorname{Re}=0.40$ ), however, it takes more than 100 minutes for it to get saturated for Run $1(\operatorname{Re}=0.0040)$.

These results show the successful application of our novel automated and efficient vision system to obtain flow and transport information in porous media. The new imaging system can be employed to get detailed insights not only in porous medium problems but also in various industrial processes where accurate automated imaging is important for analysis of the systems. 


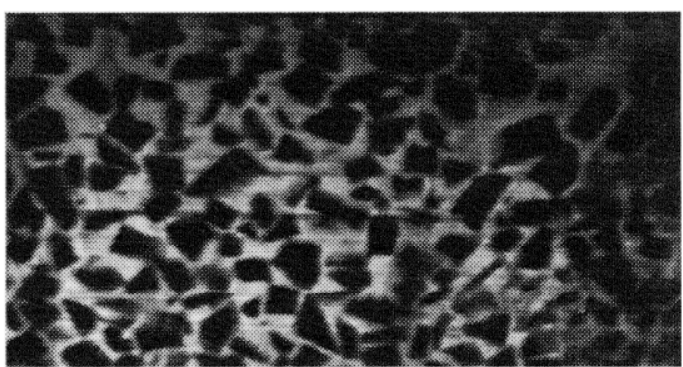

6a. Typical image slice sample at a couple of minutes after dye has been introduced to the column.

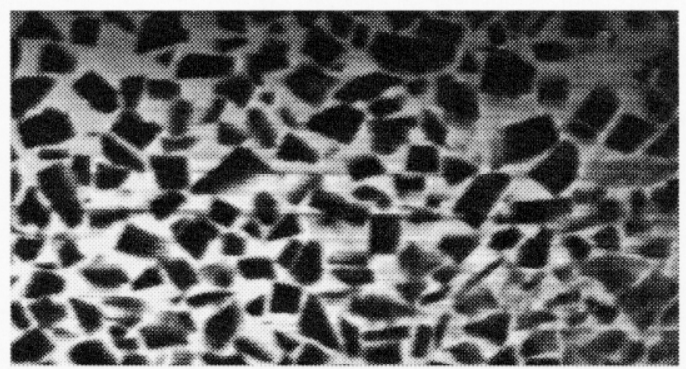

6c. Image slice after saturation of the dye.

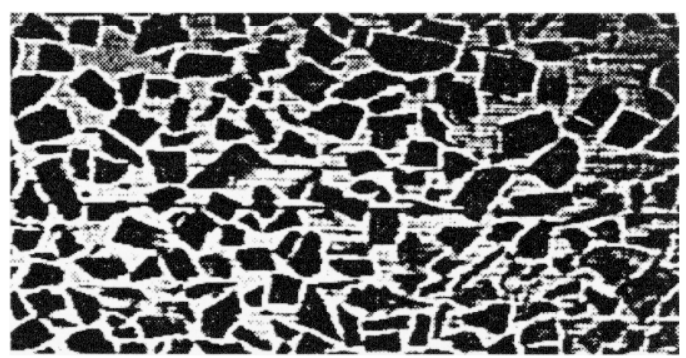

6e. Sharpening filter is applied to image resulting from subtraction of Figure $6 b$ from Figure $6 c$.

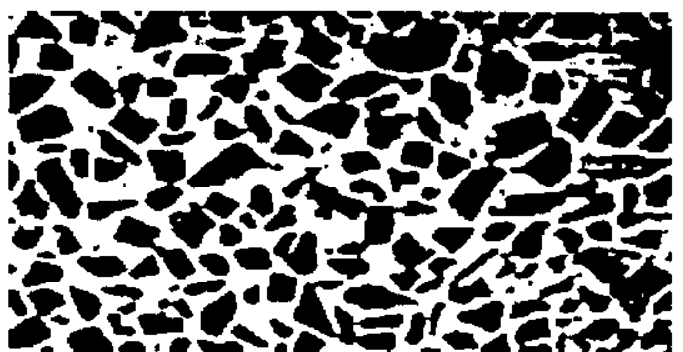

6g. Bead Image.

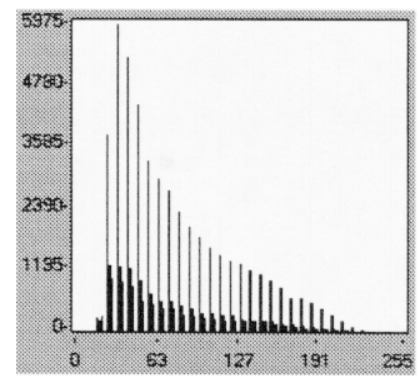

7a. Histogram of a typical image slice sample at a couple of minutes after dye has been introduced to the column.

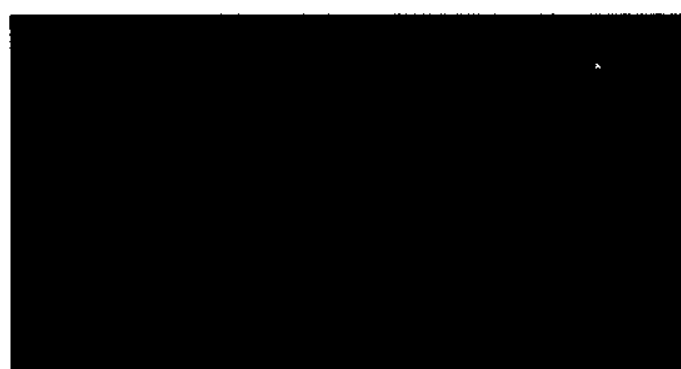

$6 \mathrm{~b}$. Image slice before the introduction of dye.

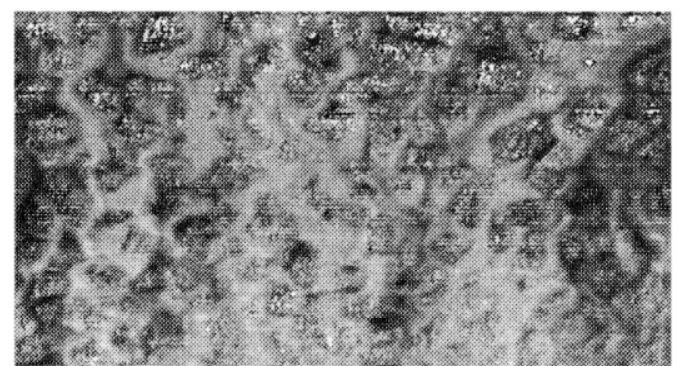

6d. Resulting image from equation (2).

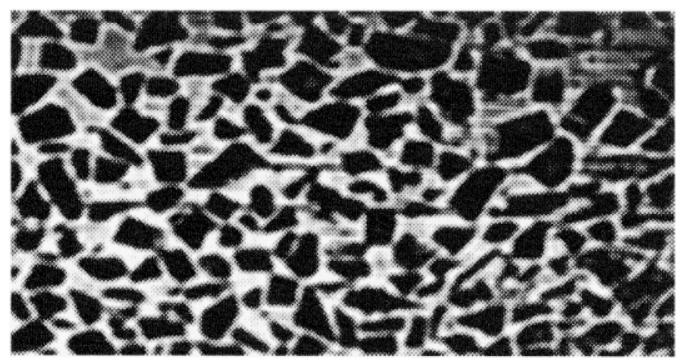

6f. Median filter is applied to the image in Figure 6e.

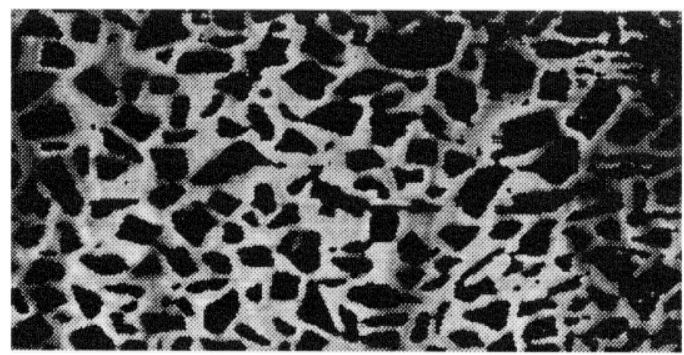

6h. Final image after all preprocessing has been completed.

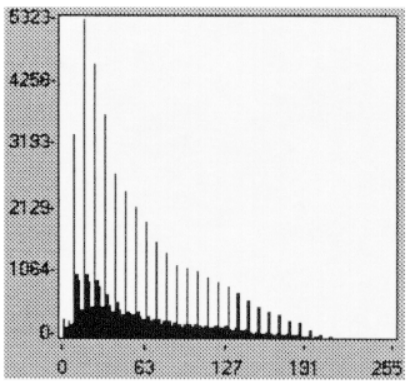

7b. Histogram of Figure $6 b$ subtracted from Figure $6 \mathrm{a}$ and the lowest pixel value is now zero.

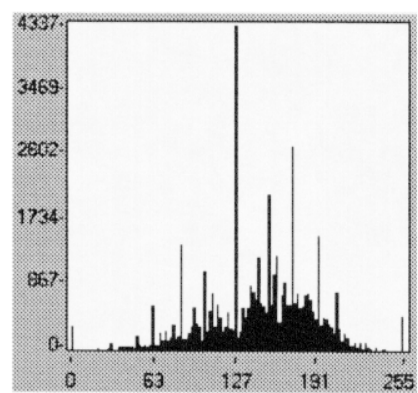

7c. Histogram of Figure 6d. Image spans the entire dynamic range of pixel values. 


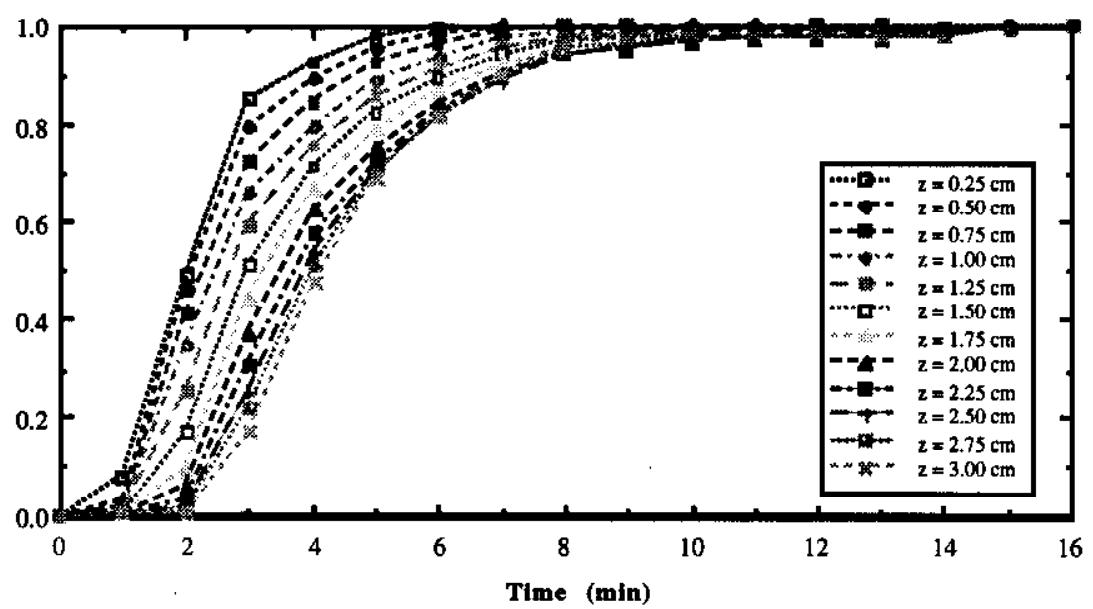

Figure 8. Distribution of cross-sectionally averaged concentration as a function of time at 12 (out of a total of 64) axial positions. Here, $z$ represents the distance from the column entrance $\left(Q=5.7 \mathrm{~cm}^{3} / \mathrm{min}\right.$, Run 5$)$.

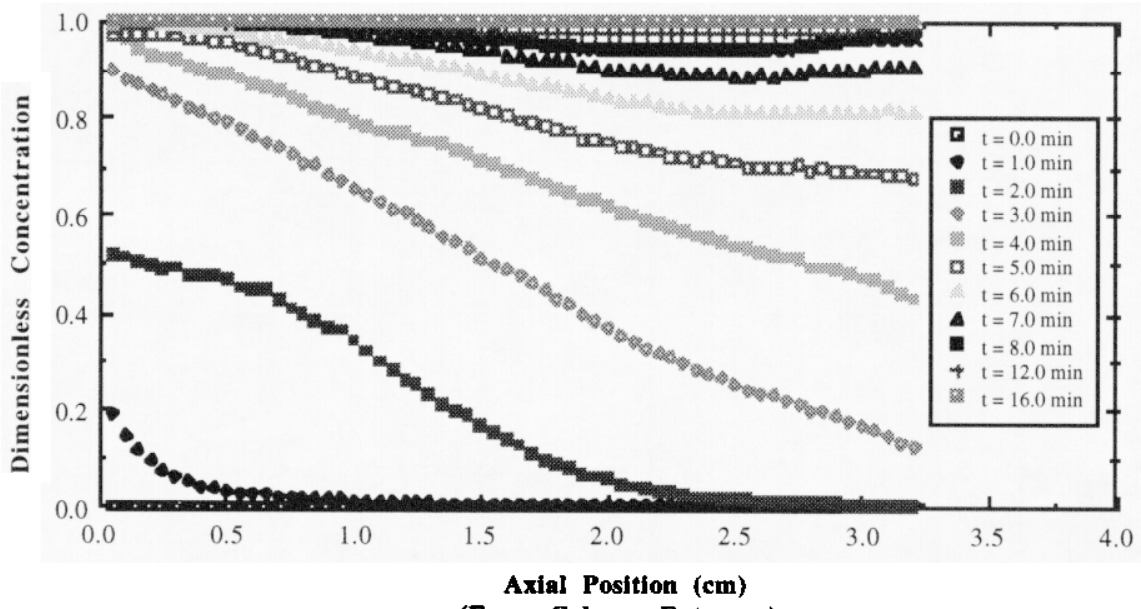

(From Column Entrance)

Figure 9. Distribution of cross-sectionally averaged concentration as a function of axial position (z) in the column at 11 different times. Data from 64 cross-sections are shown $\left(\mathrm{Q}=5.7 \mathrm{~cm}^{3} / \mathrm{min}\right.$, Run 5$)$.

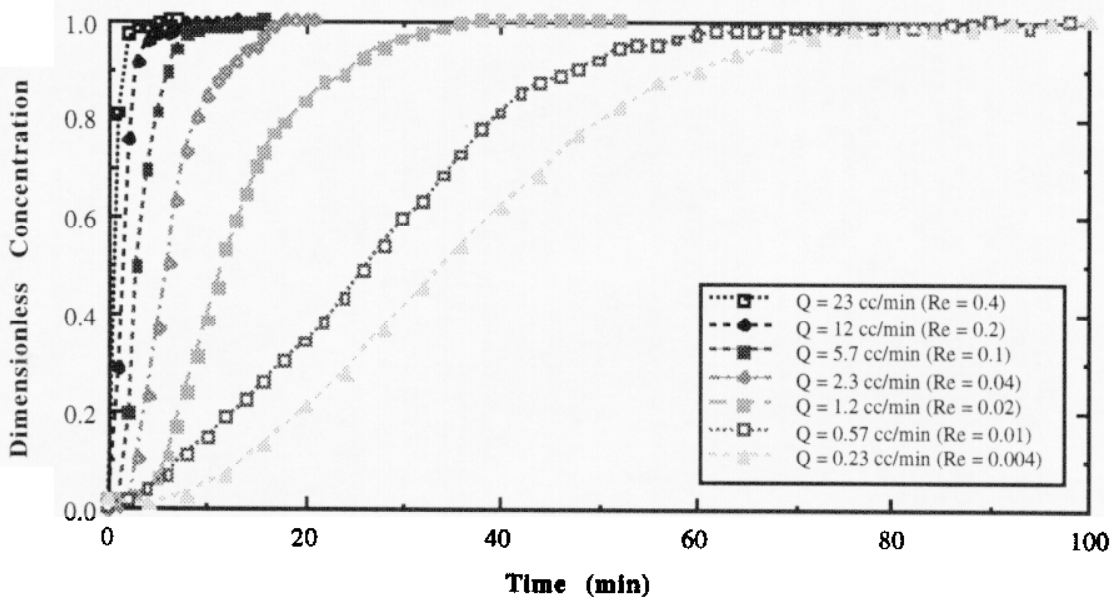

Figure 10. Distribution of volumetrically averaged concentration as a function of time for the 7 experimental runs. 


\section{CONCLUSIONS}

Development of an accurate automated image processing system has been described with applications to transport in porous media. Detailed microscopic velocity and concentration fields have been computed within the 3D volume of a porous column by using our novel automated and efficient vision system. Recorded experimental images are automatically transferred to the computer by relevant algorithms through an RS-232 and enhanced using several image processing techniques. For the velocity measurement, a sharpening filter is applied to images to detect the sharp edges of the microspheres and then followed by a binarization of the image to separate the foreground from the background in order to distinguish which pixels are microspheres. For the concentration measurements a technique using the XOR function is used to match images with the same solid phase topology and differing intensities. Then a Contrast Stretching technique is applied to images, so the images exhibit a wide range of pixel values, thus no single shade will dominate the image. Finally the Bead image technique is used to convert false meaningless pixel values produced by the Contrast Stretching technique into zeros. The image processing techniques are used successfully to obtain concentration and velocity measurements within a porous medium.

\section{ACKNOWLEDGEMENTS}

The authors would like to thank U. S. Department of Energy for funding of this project, under LDRD contract \# 95LW-026. The authors would also like to acknowledge Dr. F. Milanovich (LLNL), Dr. F. Hoffman (LLNL), Dr. A. Tompson (LLNL), Professor R. Rinker (UC Santa Barbara), and Professor S. Whitaker (UC Davis) for their valuable assistance in this research. Portions of this work were performed under the auspices of the U.S. Department of Energy by the Lawrence Livermore National Laboratory under contract number W-7405-Eng-48.

\section{REFERENCES}

1. C. E. Schwartz and J. M. Smith, "Flow distribution in packed beds," Ind. Eng. Chem. 45, 1209-1218, 1953.

2. D. Murphy, "Experimental study of low speed momentum transfer in packed beds," Ph.D. Thesis, Case Western Reserve University, 1967.

3. W. N. Musser, "Low dispersion columns for large scale chromatographic separations," Ph.D. Thesis, Case Western Reserve University, 1971.

4. K. R. Jolls and T. J. Hanratty, "Transition to turbulence for flow through a dumped bed of spheres," Chem. Engng Sci. 21, 1185-1190, 1966.

5. K. R. Jolls and T. J. Hanratty, "Use of electrochemical techniques to study mass transfer rates and local skin friction to a sphere in a dumped bed," AIChE J. 15, 199-205, 1969.

6. D. R. E. Harleman and R. R. Rumer, "Longitudinal and lateral dispersion in an isotopic porous medium," $J$. Fluid Mech. 16, 385-394, 1963.

7. R. C. Hassinger and D. U. Von Rosenberg, "A mathematical and experimental examination of transverse dispersion coefficients," Trans. Soc. Pet. Eng. J. 243, 195-204, 1968.

8. D. Klotz, K. P. Seiler, H. Moser, and F. Neumaier, "Dispersivity and velocity relationship from laboratory and field experiments," J. Hydrol. 45, 169-184, 1980.

9. N. W. Han, J. Bhakta, and R. G. Carbonell, "Longitudinal and lateral dispersion in packed beds: Effect of column length and particle size distribution," AIChE J. 31, 277-288, 1985.

10. A. Dybbs and R. V. Edwards, "A new look at porous media fluid mechanics- Darcy to turbulent," Fundamentals of Transport Phenomena in Porous Media, Martinus Nijhoff Publishers, Boston, 201-256, 1984.

11. J. L. Stephenson and W. E. Stewart, "Optical measurements of porosity and fluid motion in packed beds," Chem. Eng. Sci. 41, 2161-2170, 1986.

12. S. A. Bories, M. C. Charrier-Mojtabi, D. Houi, and P. G. Raynaud, "Non invasive measurement techniques in porous media," Convective Heat and Mass Transfer in Porous Media, S. Kakac et al. (eds.), Kluwer Academic Publishers, Netherlands, 1991.

13. S. Saleh, J. F. Thovert, and P. M. Adler, "Flow along porous media by particle image velocimetry," AIChE J. 39, 1765-1776, 1993.

14. T. Li, J. D. Seymour, R. L. Powell, M. J. McCarthy, K. L. MacCarthy, and L. Ödberg, "Visualization of flow patterns of cellulose fiber suspensions by NMR imaging," AIChE J. 40, 1408-1411, 1994.

15. J. A. Derbyshire, S. J. Gibbs, T. A. Carpenter, and L. D. Hall, "Rapid three-dimensional velocimetry by Nuclear Magnetic Resonance imaging," AIChE J. 40, 1404-1407, 1994.

16. C. D. Montemagno and W. G. Gray, "Photoluminescent Volumetric Imaging: A Technique for the exploration of multiphase flow and transport in porous media," Geophysical Research 22(4), 425-428, 1995. 
17. M. D. Shattuck, R. Behringer, G. A. Johnson, and J. Geordiadis, "Onset and stability of convention in porous media: Visualization by magnetic resonance imaging," Physical Review Letters 75(10), 1934-1937, 1995.

18. W.E. Soll, M. A. Celia, and J. L. Wilson, "Micromodel studies of three-fluid porous media systems: Porescale processes relating to capillary pressure-saturation relationships," Water Resour. Res. 29, 2963-2974, 1993.

19. W. E. Soll and M. A. Celia, "A modified percolation approach to simulating 3-fluid capillary pressure saturation relationships," Adv. Wat. Resour., 16 (2), 107-126, 1993.

20. J. Wan and J. L. Wilson, "Visualization of the role of the gas-water interface on the fate and transport of colloids in porous media," Water Resour. Res. 30, 11-23, 1994.

21. M. Rashidi, "Simulation of contaminant flow in a laboratory-scale porous system," EUROPTO, Paper No. 2504-85, Munich, June 19-23, 1995.

22. M. Rashidi, L. Peurrung, A. F. B. Tompson, and T. J. Kulp, "Experimental analysis of pore-scale flow and transport in porous media," Advances in Water Resources 19(3), 163-180, 1996a.

23. M. Rashidi, A. F. B. Tompson, T. J. Kulp, and L. Peurrung, "3D microscopic measurement and analysis of chemical flow and transport in porous media," ASME J. Fluids Eng. 118, 470- 480, 1996b.

24. M. Rashidi and E. Dickenson, "Small scale flow processes in aqueous heterogeneous porous media," ASME Fluids Engineering, FED-Vol. 239, 409-414, 1996.

25. S. Whitaker, "Diffusion and dispersion in porous media," AIChE J. 13, 420-429, 1967.

26. J. C. Slattery, "Flow of viscoelastic fluids through porous media," AIChE J. 13, 1066-1071, 1967.

27. J. Bear, "Dynamics of Fluids in Porous Media," Elsevier, New York, NY, 1972.

28. S. M. Hassanizadeh and W. G. Gray, "General conservation equations for multi-phase systems: Averaging procedures," Advances in Water Resources 2, 131-144, 1979.

29. W. G. Gray, A. Leijnse, R. L. Kolar, and C. A. Blain, "Mathematical Tools of Changing Spatial Scales in the Analysis of Physical Systems," CRC Press, Boca Raton, 1993.

30. M. Rashidi, "Non-Invasive Monitoring of Multiphase Flows," Editors: J. Chaouki, F. Larachi, and M. P. Dudukovic, Chapter 15, Elsevier Publishing Co., New York, NY, 1997.

31. M. Rashidi and S. Banerjee, S., "Turbulence structure in free surface channel flows," Phys. Fluids 31, 24912503, 1988. 


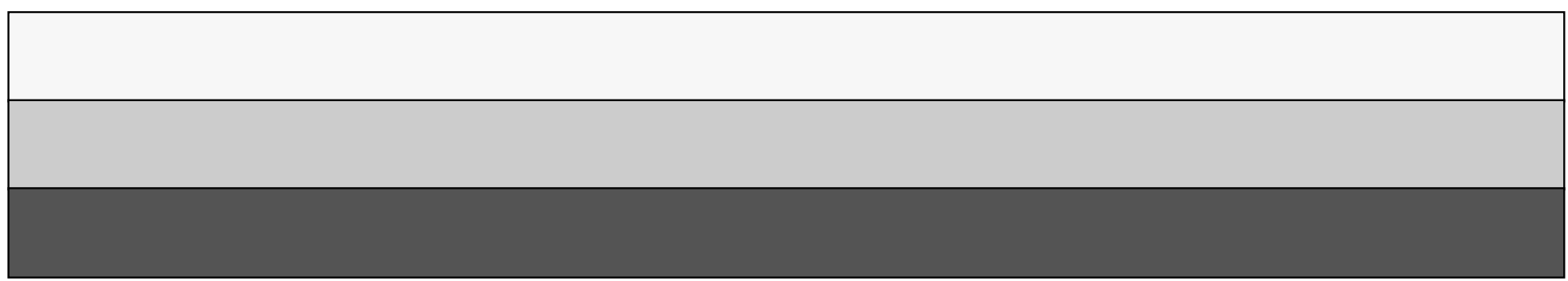

